\title{
DESIGN AND ANALYSIS OF LOW POWER HIGH-SPEED FULL ADDER BASED DETECTION COMBINATION ALGORITHM USING FINFET TECHNOLOGY
}

\author{
RAMACHANDRAN VARATHARAJAN \\ Bharath University, Chennai, India \\ Email : varathu21@gmail.com
}

\begin{abstract}
It is an efficient implementation of the Fin-Fet arithmetic circuit used in providing processor making analytical tasks. The complementary metal-oxide-semiconductor leakage current depends on the driving capacity, which is delayed from the compression circuit. This method is based on any real-time digital signal processing application, where the drive current must be high. The conventional design will have a low-efficiency supply voltage with low capacitance; even when operating, the circuit speed will be faster, and the delay will be less. The method add-on structure is designed to select or transfer gates from input data to output. The proposed 10T full adder and adder is given in and respectively. Low operation Very low-level integrated circuit, capable of low power consumption, which has become an essential standard operation for the design of energy-efficient electronic products, has become the primary function and compact device design, once again possible. The coefficient plays a significant role in planning energy-saving processors and determining processor performance. The multiplier coding booth method is used to rearrange the input bits to reduce device usage. Changing the equal position of a given booth is the behavior of the booth decoder. Booth decoder increases the range of different zero's. Therefore, the switching behavior is reduced to reduce the power consumption of the design further.
\end{abstract}

Keyword: Fin Field-effect transistor (Fin FET), CMOS (complementary Metal-oxidesemiconductor), Full adder

\section{INTRODUCTION}

\section{FIN FET TECHNOLOGY}

In using different functions of coordinate circuits, the appendix expands its capabilities beyond a few nanometers and loses it. Much work has been done from writing to implementing Fin-Fet based courses. The facts show that the primary constraints in using CMOS (Corrosive Metal Oxide Semiconductor) semiconductors are high power consumption and high spillage current. Scaling has less outrageous channel effects, which makes it harder to control. Metal oxides increase the leakage of current and double energy consumption. This problem can be solved by using the multi-door MOS-FET innovation, which reduces these barriers by giving a powerful silicone body and powerful command over various electrified coupled entry gates 


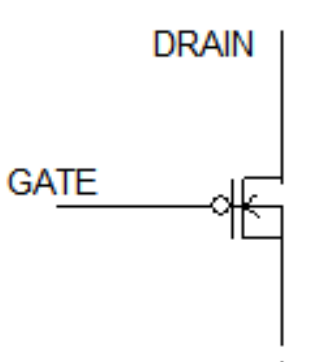

SOURCE

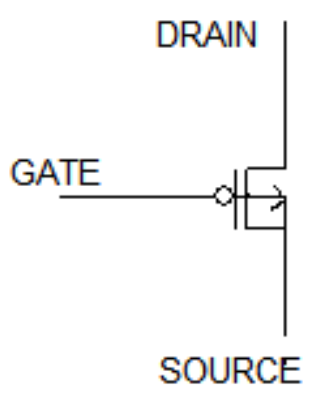

Figure 1 Symbol of Fin-Fet Technology

\section{CMOS (Complementary metal-oxide-semiconductor)}

CMOS systems include system, pull-down system PMOS semiconductor, and a pull-up. On the possibility that the PMOS semiconductor exceeds the high rational "1" and the weak logical "0", the NMOS semiconductor moves the high balanced "0" and the soft intelligent "1" to the next level. The output of the system is exhausted, and the voltage level does not drop (due to human body effects, the plan builds an unlimited voltage drop). Significant CMOS Advanced door power consumption is dynamic, static, and short out force consumption. The actual force consumption of the CMOS circuit depends on the boundaries, for example, the magnitude of the gravitational power voltage, clock repetition, and dynamic exchanging hubs. Reducing each of the above limitations will reduce power consumption

\section{PREVIOUS RESEARCH WORK}

Low-power and low-voltage circuits are a large scale integration. The other is more basic, and, in most cases, processors and multitask performance are used [1]. The modified booth multiplier is one of the various methods of signature multiplication. It is usually used as a speed coefficient. Here, we have created a multiplier booth modification that uses gate diffusion input technology using traditional methods for low-power 8-bit [2].

Using some nanometer technology to compare and analyze all transistor-free and power delay designs [3]. Encoder and decoder include the partial output generator for the multiplier, the 1-bit (half and full) adder, and the final adder. The booth coefficient identifies the small high-speed multiplication circuit by two high-frequency signals [4]. Large-scale integration of high-speed design, area-effective, and low-power of high-architecture require an efficient arithmetic processing unit [5]. Design key building blocks, multiplication and signal processing, and a system that uses each input and output source. Displays statistical data of 
multiplication operations performed from specified values by signal processing of multiple processors and digital techniques. Therefore these operations require more implementation time [6].

The level of integration continues to grow and is implemented in an integrated design in line with more advanced signal processing systems. This booth decoder increases the number of multipliers to zero [7]. The booth coefficient contains the booth decoder and is specified in the input booth equivalence of the re-encoder [8]. This design can reduce power consumption, thereby reducing the number of switching operations [9]. When the input coefficient is in the range corresponding to zero, the input bit coefficient determines the variable behavior of the component, or the declaration column must be deactivated [10]. The speed of the factor determines the speed of the processor.

Therefore, faster multipliers are needed [11]. The function of dynamic domino logic gates on the metal oxide semiconductor of complementary static gates is that they are quicker and require less area. However, the total power consumption is higher than that of static gates [12]. This is a very high-coefficient speed booth obtained by partial production. The coefficient appears to have halved algorithm required partial output due to the speed of the partial factor [13]. The booth coefficient consists of a three-component encoder, a partial output generator, and an adder circuit [14]. When two numbers multiply the $\mathrm{N}$-digit coefficient and the $\mathrm{N}$-digit coefficient, each fraction has an M-digit factor with the N-digit ratio. This occurs, and the multiplication of the coefficient forms a circuit [15].

\section{MATERIALS AND METHOD:}

Traditional, complementary metal oxide with complete adder unit. It has ten transistors in a 1-bit entire adder unit. The additional metal-oxide-semiconductor design style is not an active area of the door with excellent performance. Therefore, care must be taken when selecting a static logic style to execute a logic function. The pass transistor logic model is the basis for a standard method known for implementing specific circuits such as flip flop circuits, and exclusive OR. The full adder's standard static complementary is based on the traditional metal oxide architecture and the classic design of conventional pull-up and pull-down resistors, which provides transistor total swing output and excellent driving performance. The algorithmbased controller is designed for a fast and accurate response. The method offers better control 
via a single-loop control system. The relay feedback technique is used for the tuning controller, and this method can be extended to the auto tune of the cascade controller system. The traditional on-off relay oscillation has been continued for the single loop feedback controller. The method presented in the application is used to obtain its parameter for tuning of cascade controller with minimum computing complexity resources, and the delay is calculated and analyzed for the different supply voltage.

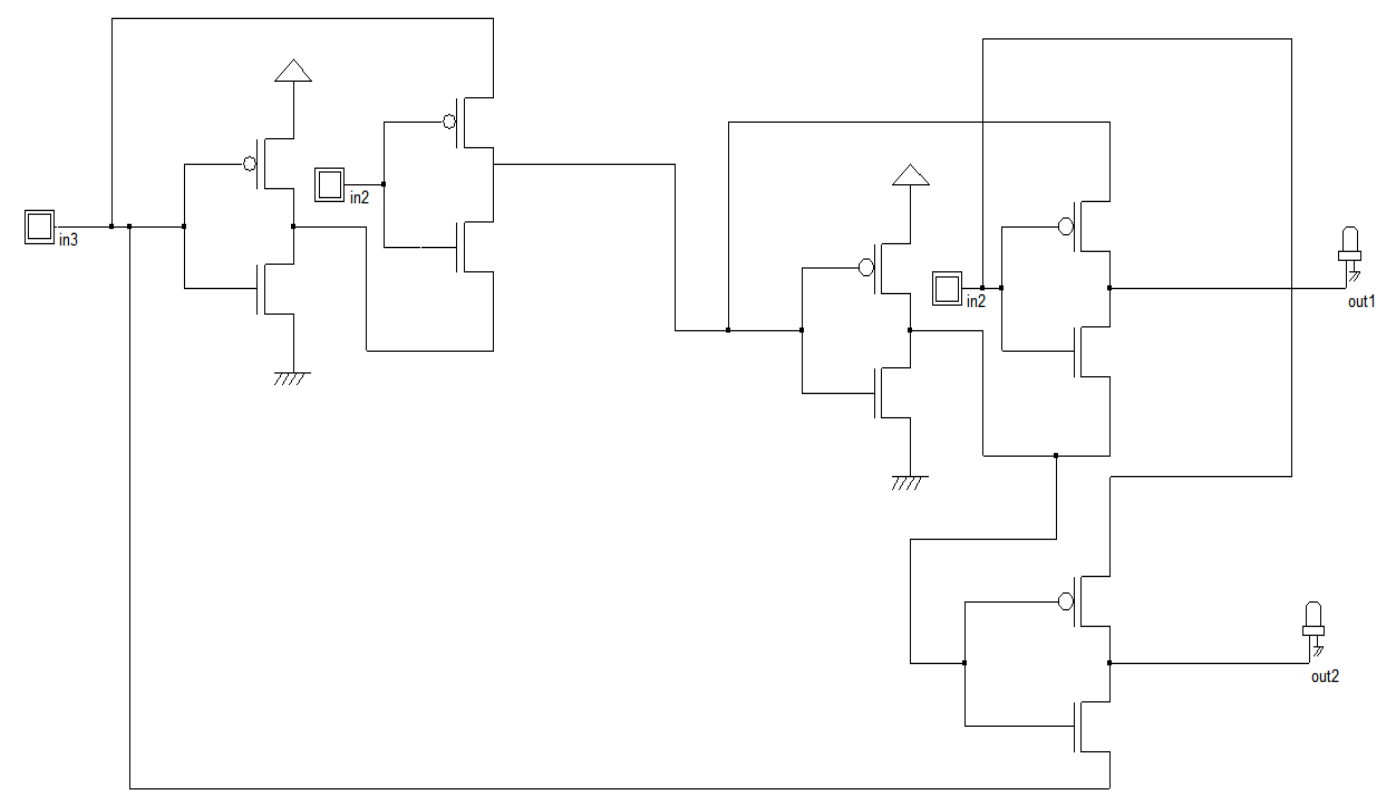

Figure: 2 Schematic Diagram 10T full adder using DSCH

\subsection{WORKING PRINCIPLE}

Figure 2 shows the appearance DSCH of an original structure largely depends on the unit being added. Due to the extensive utilization of this functionality in number-crunching capabilities, many experts have had the late-suggested enthusiasm to propose some distinctive rational styles to make 1-piece whole merging units a reality. To perform no juggling operations, gadgets do not lose any power by working at very-low-frequency reference books, although they do devote a lot of effort to complete. Force building deferral is used for significant level correlation between different construction square plans. Interest in low-power, considerable scope coordinated circuits continues to grow and can be met at various architectural levels, including engineering, channel, design, and policy innovation levels. At the circuit configuration level, a lot of brilliance can be left out by choosing the rational style used to run the mixing circuit. The interest and predominance of versatile electronic goods are bidding creators to maintain lower terrains, faster speeds, longer battery life, and higher volatile quality. Force utilization and deferral frameworks topped the list of assets. 


\subsection{FULL ADDER}

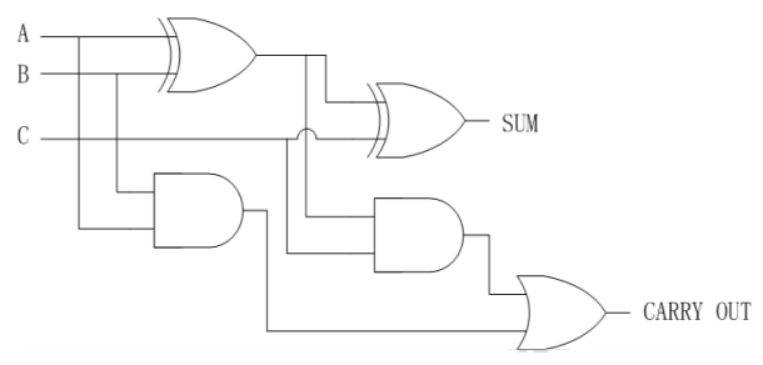

\section{Figure 3 Schematic Diagram for the Full adder}

Figure 3 shows Adder is a digital circuit that contains numbers. Produces two yields, for example, Half Adder Add and Carry. It is applied to two data terminals to create a measure of $\mathrm{XOR}$, and the AND access path is used to give two data rural objects. The whole full adder takes three 1-piece numbers, called two operands and one denoted in bits. At that point, it produces a 2-piece yield, called the creation motion. First, four to four promotions were made using the traditional full adder. Features custom, corresponding metal-oxide-semiconductor complete ads base, and top advanced flagging circuits. It consists of 10 semiconductors and induces high power consumption using 180-nanometer Ace tools and the schematic outlines of the 10-semiconductor full adder. The four-tree coefficient appears in the 10-semiconductor adder scheme.

\subsection{ANALYSIS OF LOW POWER HIGH SPEED}

The beat generator is a flip-flop, which can be divided into a few flip-failures to reduce power consumption and chip area. With the inclusion of parallel circuits, the speed is practically constant, while the overhead of the information signal exchange point is exceptionally low. This strategy shows that the flip-flop speaker has a clock-gated acceptance structure, a variable innovation intended to improve performance, work speed, and achieve higher power productivity. Talk about reducing energy by reducing charisma. By reducing the power in backup mode, the creator reports a decrease in power consumption. Reduce backup power consumption and power consumption-force sparring strategy using single triggers by shut circle voltage scaling. Force sparing rate is higher when the gadget loses position, but we have examined and broken the door size of the Fin-Fet device. 
TABLE 1 TRUTH TABLE OF FULL ADDER

\begin{tabular}{|c|c|c|c|c|}
\hline \multicolumn{3}{|c|}{ INPUT } & \multicolumn{2}{c|}{ OUTPUT } \\
\hline A & B & C & SUM & CARRY \\
\hline 0 & 0 & 0 & 0 & 0 \\
\hline 0 & 0 & 1 & 1 & 0 \\
\hline 0 & 1 & 0 & 1 & 0 \\
\hline 0 & 1 & 1 & 0 & 1 \\
\hline 1 & 0 & 0 & 1 & 0 \\
\hline 1 & 0 & 1 & 0 & 1 \\
\hline 1 & 1 & 0 & 0 & 1 \\
\hline 1 & 1 & 1 & 1 & 1 \\
\hline
\end{tabular}

The power consumption of high-speed portable devices is very important. Today, lowpower design and high-speed VLSI systems are becoming more and more important for the rapid growth of portable devices. Power consumption and performance of the circuit are major trade-off factors for low-power VLSI design. The implementation capability of an arithmetic circuit affects the performance of the entire integrated circuit and the digital signal processor. Implementing dedicated algorithms significantly increases the execution efficiency of the arithmetic circuit and affects the performance of the processor and the integrated circuit dedicated to the application. Contains the sum and value of the two inputs generated by the complete addition.

It can be combined with other complete add-ons or your work. Full adder is still one of the main centers of research for many years, and it is one of the fundamental pillars of circuit applications. Different logic styles each have their advantages and barriers. Consider more straightforward and more reliable implementations with lower power requirements, but chip area requirements compare to their dynamic counterparts. It became more significant than usual. These models use different logic style features to enhance the overall performance of the full adder

\subsection{DETECTION COMBINATION ALGORITHM}

Duplicate signature of algorithmic number is a more complicated process. By unsigned 
reinforcement, there is no compelling reason to consider the reference to the amount. Although a similar method cannot be applied because the number identified in the identified duplicate is in the compliment structure, it gives the wrong result when increased when it is practically equivalent to the unsigned photocopy. Hence here comes the counting of the booth. The counting of the stall oversees the forecast of the final product. When duplicating, the coefficient is called when moving a series of 0 seconds.

When reinforcing, activity is required at each end of the $1 \mathrm{~s}$ range in the ratio. We need to add or subtract at positions of the coefficient that change from 0 to 1 or from 1 to 0 . In the accompanying stream format, $\mathrm{b}=$ multiplier, $\mathrm{a}=$ multiplier, $\mathrm{m}=$ product. Right now, we need twice as many pieces in our item here. The left-hand side of our operations of both the coefficient and the coefficient is a symbol that is constant and not used as an attribute of value. What Operand multiplies at that point and what must be reproduced. In the case where one operation and both are negative, at that point, they are both spoken in an ancillary structure. Start with the item that has the module in the organization of the extra driving zero bits. Check the LSB present and the last LSB of the article to find the number-crunching functionality. Add 0 as the first LSB, which is the first pass framework count.

STEP I. If they are 01, find the value of P + A. Ignoring any overflow.

STEP-II. If they are 10, find the $\mathrm{P}+\mathrm{S}$ values. Ignore anything Overflow.

STEP III. If they are 00, do nothing. Use P directly. The next step.

STEP IV. If they are 11, do nothing. Use P directly. The next step.

TABLE 2 CALCULATION OF FRONT PROPAGATION (BP) ALGORITHM

\begin{tabular}{|l|l|l|l|l|}
\hline xi & Xi-1 & Qi-1 & Multiplier Value & Condition \\
\hline 0 & 0 & 0 & 0 & Zero's string \\
\hline 0 & 1 & +1 & +1 & Ending string 1s \\
\hline 1 & 0 & -1 & -1 & $\begin{array}{l}\text { Beginning } \\
\text { string 1 }\end{array}$ \\
\hline 0 & 1 & & & String 1s \\
\hline
\end{tabular}




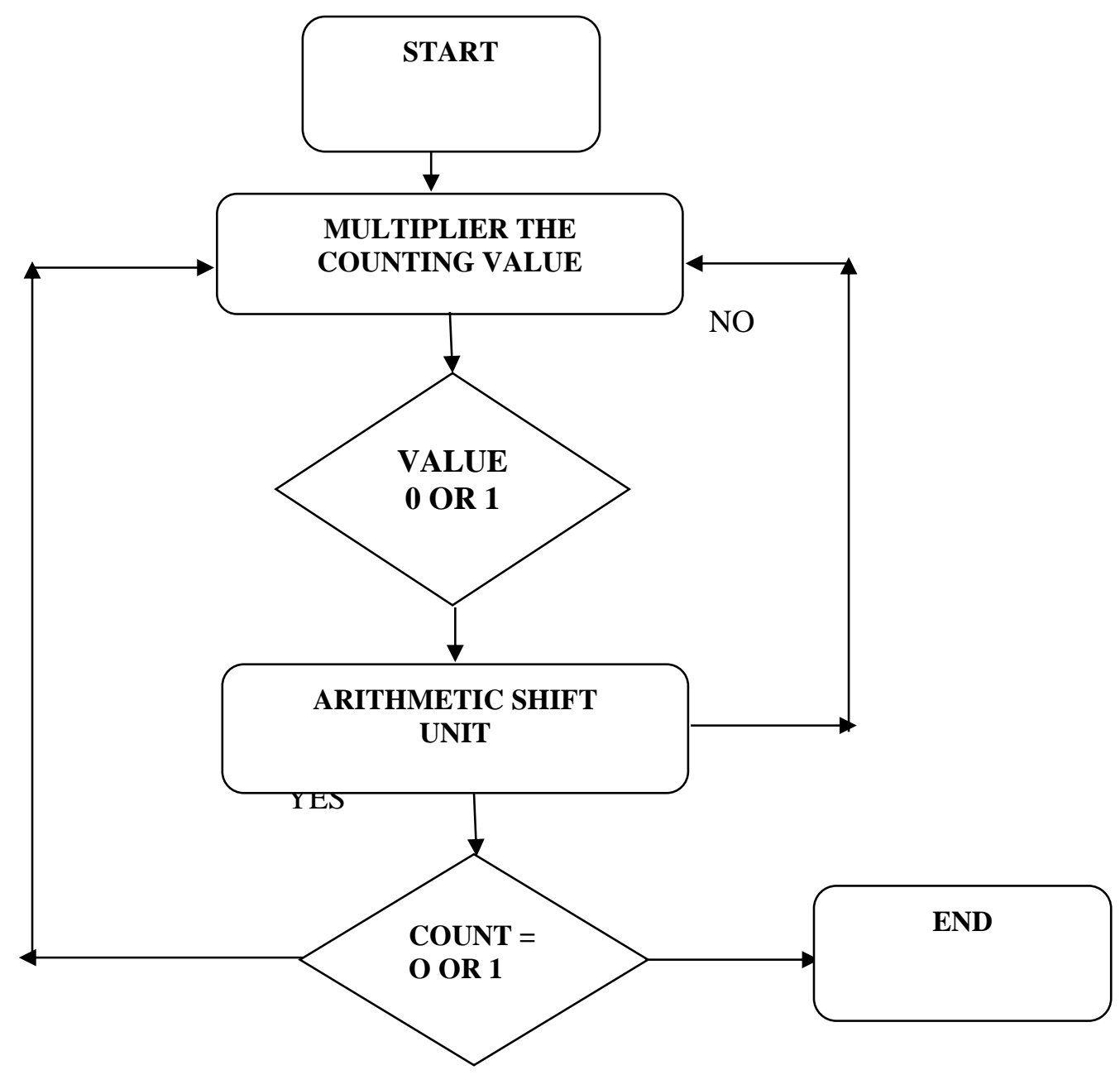

Figure 4 Flow chart of the Front propagation algorithm

\section{RESULT AND DISCUSSION:}

\subsection{SIMULATION RESULT}

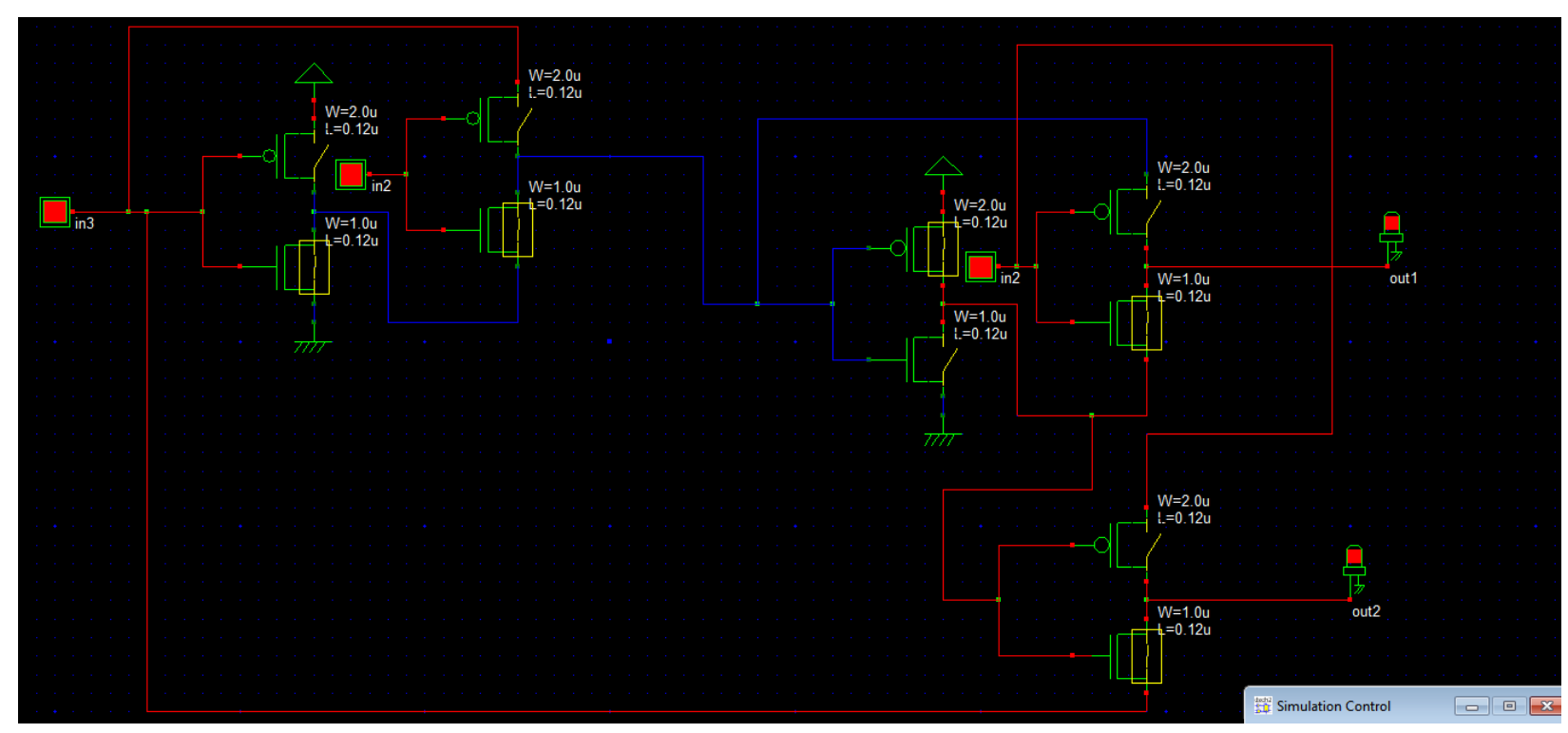

Figure 5 Schematic Diagram DCSH using 10T Full adder 
When VDD $=1 \mathrm{v}$, compared to Conventional CMOS, the proposed 10T adder achieves power savings of $41.65 \%$. It is also observed that compared to the $14 \mathrm{~T}$ adder.

\section{OUTPUT WAVEFORM}

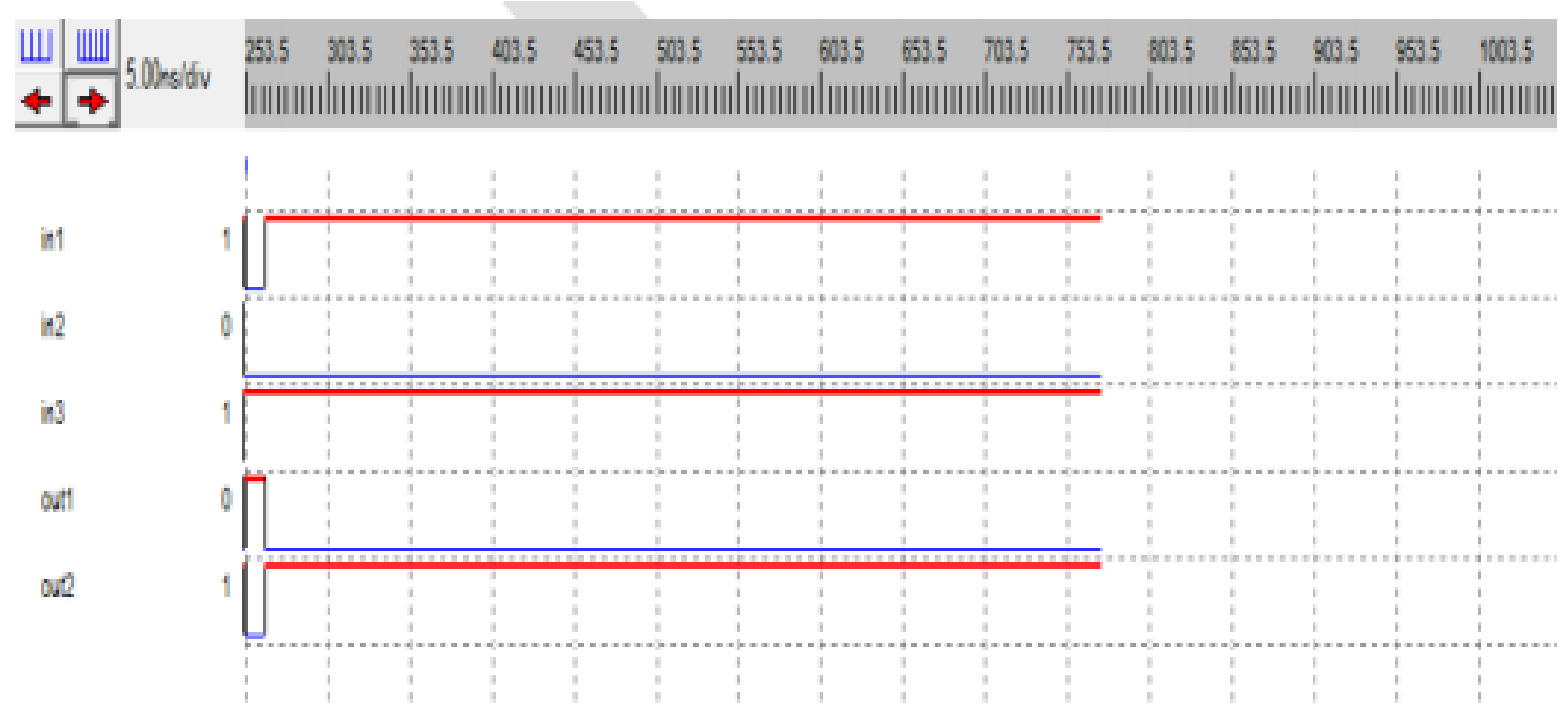

Figure 6 Output DCSH wave source of 10T full adder

Figure 6 shows plots of supply voltage versus Power dissipation of CMOS adder, 10T adder, and proposed 10T adder for supply voltages. It is observed from the fig that the reduction of supply voltage VDD will abruptly decrease the average power consumption of the circuit.

\section{SIMULATION OUTPUT}

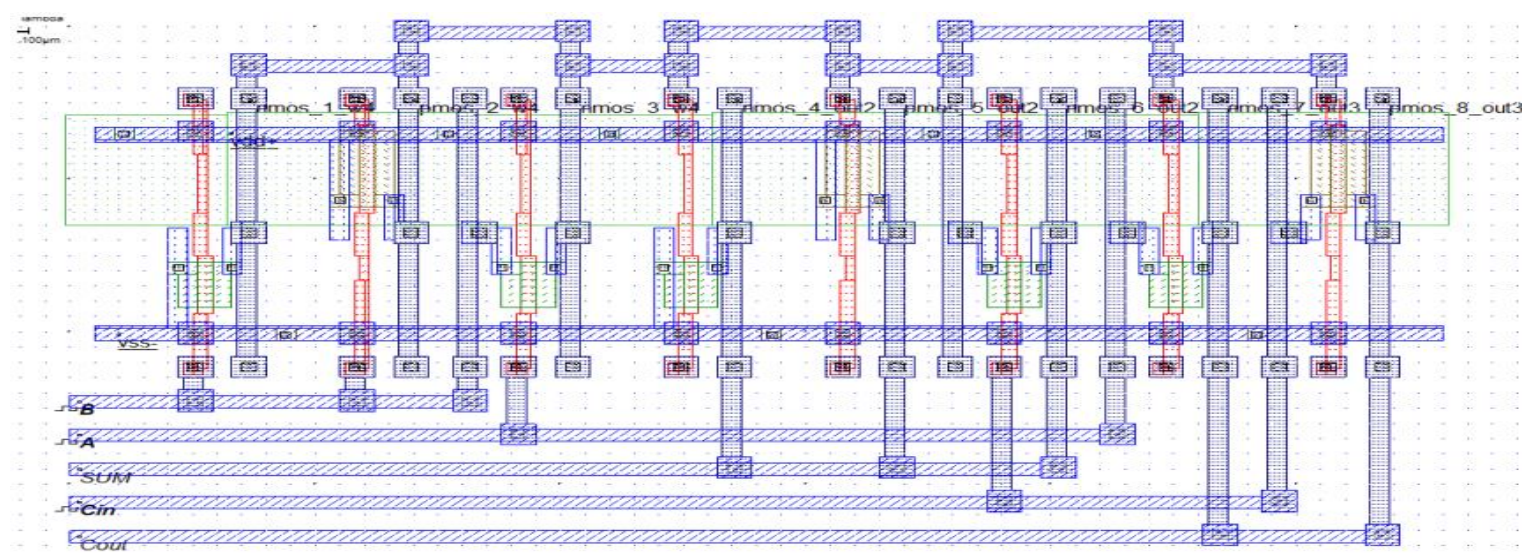

The Layout setup of the XOR entryway format incorporate dissemination, $\mathrm{N}$ dispersion, contact cutting, metal, and are utilized for NMOS and PMOS semiconductors. The design comprises of XOR circuits and is because of time deferral and force utilization. Contrasted with the XOR door, the time is exceptionally little. Subsequently, the base number of 
semiconductors with expanding and time is a large format plan with a $32 \mathrm{~nm}$ procedure.

\section{CHARACTERISTICS OF THE LAYOUT}

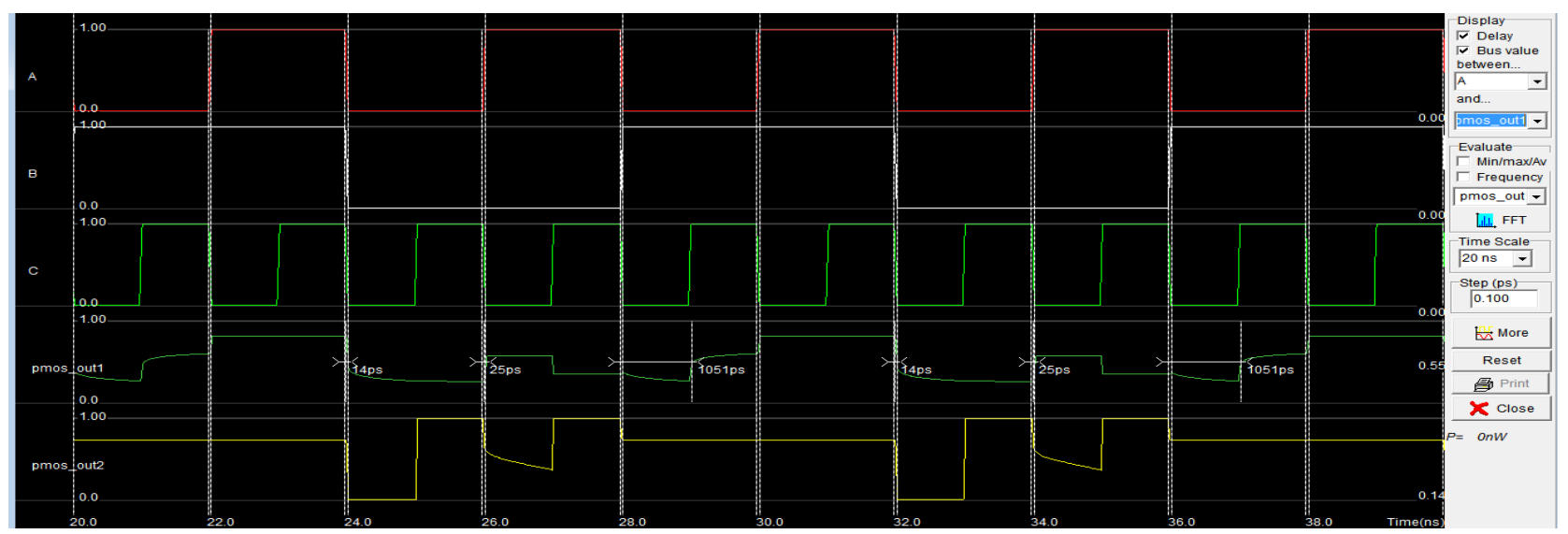

Format characteristics due to current and voltage at the channel consider channel source voltage VDS and current Ids. Due to the current characteristics, it is equally constant and varies with voltage difference. Since it is ably reversible with the reproduction of XOR door time. Consequently, the postponement is short.

\subsection{TABULATION}

\begin{tabular}{|l|l|l|l|}
\hline ADDER TYPE & $\begin{array}{l}\text { NO OF } \\
\text { TRANSISTOR }\end{array}$ & DELAY & Input Source \\
\hline Half Adder & & 104 & 0.54 \\
\hline Full Adder & 8 & 230 & 6.00 \\
\hline $\begin{array}{l}\text { Existing } \\
\text { Method }\end{array}$ & $14 \mathrm{~T}$ & 520 & 2.36 \\
\hline Proposed Method & $10 \mathrm{~T}$ & & 1.20 \\
\hline
\end{tabular}

Figure 7 Tabulation for 10 T Full adder 


\subsection{TABLE CHART}

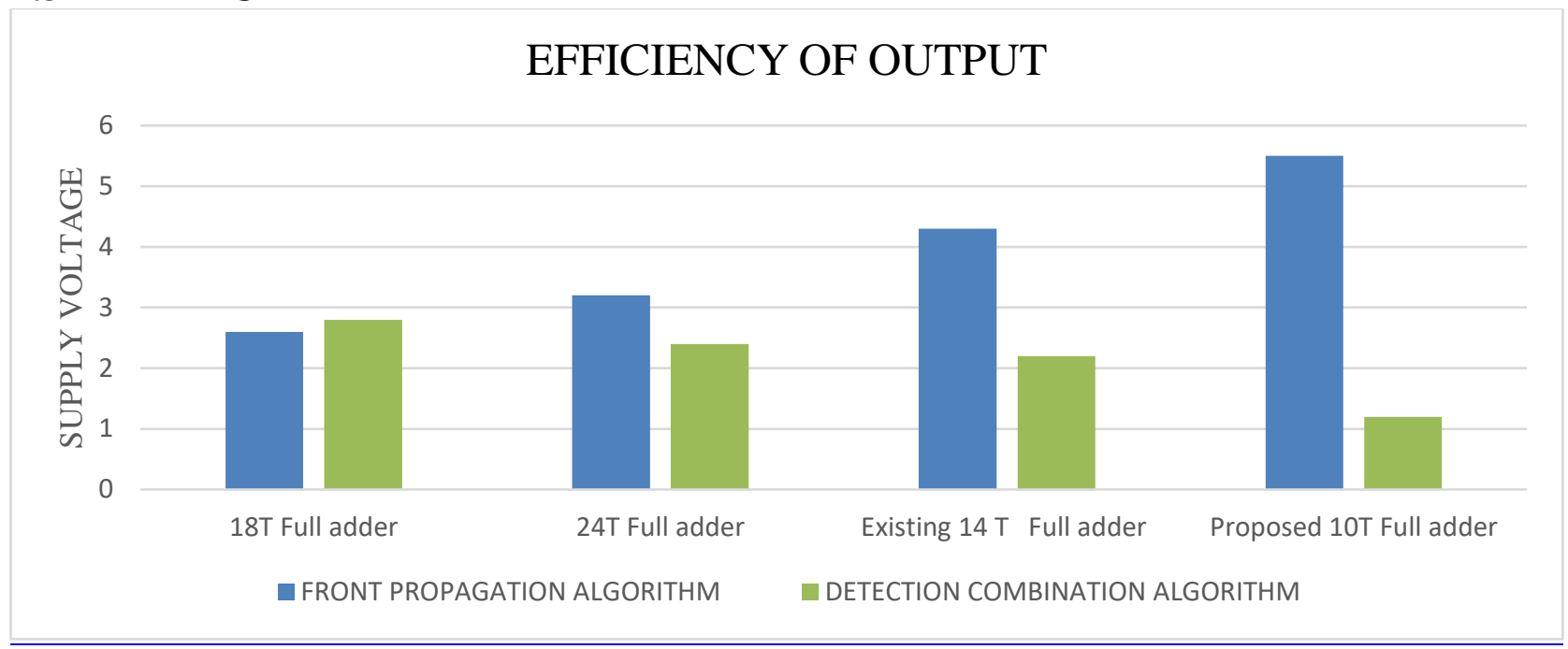

Figure 8 Table calculation for Power Dissipation

Figure 8 shows plots of supply voltage versus delay of CMOS adder, 10T adder, and proposed $10 \mathrm{~T}$ adder for supply voltages $\mathrm{Vdd}=1 \mathrm{~V}, \mathrm{Vdd}=0.75 \mathrm{~V}$, and $\mathrm{Vdd}=0.5 \mathrm{~V}$. It is observed from the reduction of supply voltage VDD will increase the propagation delay of the circuit.

\section{CONCLUSION}

In this work, the power and Time delay efficiency of the entire connection with semiconductors using the proposed gadgets XOR circuit. The highlights of the proposed whole adder circuit due to the intensity consumption are against the complete adder circuit before the exit. Full adder shows better yield banner power utilization with reduced semiconductor materials superior performance at lower speeds and higher speeds. Force gating mechanism minimizes the leakage current in backup mode. Different boundaries are observed at various voltage supplies.

\section{REFERENCE}

1. Nataliya Chaitali, "Performance Comparison of carrying Save Adder at $180 \mathrm{~nm}, 90 \mathrm{~nm}$ and 45nm CMOS Technology" International Journal of Advanced Research in Computer Engineering \& Technology. Volume 5, Issue 5.

2. M. Kiran Kumar, "a design of low power modified booth multiplier," international journal of current engineering and scientific research, volume-5, issue-4,

3. Narmada Gupta, "Power-Aware \& High-Speed Booth Multiplier based on Adiabatic Logic," International Journal of Innovations in Engineering and Technology (IJIET), 
4. R Kalaimathi1, "A Survey on Area Efficient Low Power High-Speed Multipliers," International Journal for Research in Applied Science \& Engineering Technology, Volume 5 Issue XI 2017.

5. Jasbir Kaur, "FPGA Implementation of 4-Bit Multipliers", International Journal of Computer Science and Technology.

6. Prabhu, A. S., \& Elakya, V. "Design of modified low power booth multiplier." International Conference on Computing, Communication, and Applications.

7. Govern, D., \& Amonkar, A. "Design and implementation of high speed modified booth multiplier using hybrid adder." International Conference on Computing Methodologies and Communication (ICCMC).

8. Singh, M., Maurya, A. K., Singh, S. "6×6 booth multiplier implemented in modified splitpath data-driven dynamic logic". Students Conference on Engineering and Systems.

9. Rajawat, A., \& Marwah, A. "GDI implementation of low power modified booth multiplier." 2016 Symposium on Colossal Data Analysis and Networking (CDAN)".

10. Aravind Babu S, Babu Ramki S, "Design and implementation of high speed and high accuracy fixed-width modified booth multiplier for DSP application." International Conference on Advances in Electrical Engineering.

11. Saravanan, S., \& Madheswaran, M. "Design of Hybrid Encoded Booth Multiplier with Reduced Switching Activity Technique and low power $0.13 \mu \mathrm{m}$ adder for DSP block in wireless sensor node". International Conference on Wireless Communication and Sensor Computing

12. Sridevi, B., Gangadevi, B., "Modified booth multi-precision multiplier with scalable voltage and frequency units." IEEE International Conference on Computational Intelligence and Computing Research.

13. Rao, M. J., \& Dubey, S. "A high speed and area-efficient Booth recoded Wallace tree multiplier for fast arithmetic circuits." Asia Pacific Conference on Postgraduate Research in Microelectronics and Electronics.

14. He, W.-Q., Liu, C.-Y., Liu, W.-Y., \& Chen, Y.-H. ."A high accuracy fixed-width Booth multiplier using select probability estimation bias". International Conference on Information Science and Technology.

15. Rakshitha, P., Bindu, A., Kumar, B. V. P., Rashmi, S. B., \& Yellampalli, S. S. "Design of $60 \mathrm{GHz}$ CMOS power amplifier to improve power added efficiency". International Conference on Smart Grids, Power and Advanced Control Engineering (SPACE).2017 